\title{
Discriminative Stimulus Effects of 3,4- Methylenedioxymethamphetamine and Its Enantiomers in Mice: Pharmacokinetic Considerations
}

\author{
William E. Fantegrossi, Naoki Murai, Brian Ó. Mathúna, Nieves Pizarro, and \\ Rafael de la Torre
}

University of Arkansas for Medical Sciences, College of Medicine, Department of Pharmacology and Toxicology, Little Rock, Arkansas (W.E.F.); Yerkes National Primate Research Center, Division of Neuroscience, Atlanta, Georgia (W.E.F., N.M.); and L'Institut Municipal d'Investigació Mèdica-Hospital del Mar, Human Pharmacology and Clinical Neurosciences Research Group, Neuropsychopharmacology Program, Barcelona, Spain (N.M., B.Ó.M., N.P., R.d.I.T.)

Received January 6, 2009; accepted March 9, 2009

\begin{abstract}
3,4-Methylenedioxymethamphetamine (MDMA) is a drug of abuse with mixed stimulant- and hallucinogen-like effects. The aims of the present studies were to establish discrimination of $S(+)-M D M A, R(-)-M D M A$, or their combination as racemic MDMA in separate groups of mice to assess cross-substitution tests among all three compounds, to determine the time courses of the training doses, to assess pharmacokinetic variables after single injections and after cumulative dosing, and to define the metabolic dispositions of MDMA enantiomers and their metabolites. All three forms of MDMA served as discriminative stimuli, and with the exception of $R(-)$-MDMA in mice trained to discriminate the racemate, compounds substituted for one another. The onset of interoceptive effects for $S(+)$ MDMA and racemic MDMA were faster than for $R(-)$-MDMA, and the duration of discriminative stimulus effects was shortest
\end{abstract}

for $R(-)$-MDMA. $S(+)$-MDMA and its metabolites were found in higher concentrations than $R(-)-$ MDMA and its metabolites after a bolus dose of racemic MDMA. The $N$-dealkylation pathway is favored in mouse plasma with MDA as the main metabolite formed. Cumulative doses of MDMA lead to higher plasma concentrations compared with an equivalent single dose. 3,4Methylenedioxyamphetamine (MDA) concentrations are lower after the cumulative dose compared with the single dose, which, coupled with the nonlinearity observed in MDMA pharmacokinetics after increased doses of racemic MDMA, suggests autoinhibition (or saturation) of MDMA metabolism in mice. In total, these studies suggest that the discriminative stimulus effects of racemic MDMA are perhaps driven by accumulation of $S(+)-M D M A$ and $S(+)-M D A$ in the mouse.
Phenethylamine is a ubiquitous backbone structure in endogenous biochemicals and various drugs of abuse. Oxygenated substituents on the phenyl ring typically produce hallucinogenic drugs of the mescaline type, whereas side-chain modifications often result in stimulant drugs of the amphetamine type (Glennon, 1989). Recent interest has focused on a

This work was supported by the National Institutes of Health National Institute on Drug Abuse [Grants DA020645, RR020146, DA017987]; the Generalitat de Catalunya-Comissió Interdepartamental de Recerca i Innovació Tecnològica, Barcelona, Spain [Grant 2005SGR00032]; the Spanish Network on Addiction Disorders [Grant FIS-RTA RD06/0001/1009]; and the Yerkes Base [Grant RR00165].

Article, publication date, and citation information can be found at http://jpet.aspetjournals.org.

doi:10.1124/jpet.109.150573. third class of substituted phenethylamines, the methylenedioxy congeners, which have come to be known as "entactogens" (Nichols, 1986). Included within this group of compounds are 3,4-methylenedioxymethamphetamine (MDMA) and its analogs, all of which are club drugs often sold under the name of "ecstasy" (Cole et al., 2002). MDMA and its analogs have chemical properties common to both the central stimulants and the hallucinogens and some unique properties not shared by these more traditional drugs of abuse; thus, it has been argued that these methylenedioxy compounds represent a unique pharmacological class (Nichols, 1986; Nichols and Oberlender, 1990). It is important that both the $S(+)$ - and $R(-)$-enantiomers of MDMA are active in vivo but may differ in terms of their pharmacological (Fante-

ABBREVIATIONS: MDMA, 3,4-methylenedioxymethamphetamine; MDA, 3,4-methylenedioxyamphetamine; HMMA, 4-hydroxy-3-methoxymethamphetamine; HMA, 4-hydroxy-3-methoxyamphetamin; HHA, 3,4-dihydroxyamphetamine; DOM, 1-(2,5-dimethoxy-4-methylphenyl)-2-aminopropane; IMIM, Institut Municipal d'Investigació Mèdica; TO, time-out; FR, fixed ratio; $C_{\max }$, maximum serum concentration; $t_{\max }$, time to reach maximum serum concentration; AUC, area under the curve; $K_{\mathrm{e}}$, fractional change in drug concentration per unit time; MTP, $(R)-(-)-\alpha$-methoxy$\alpha$-trifluoromethylphenylacetyl derivative; TMS, trimethylsilyl derivative. 
grossi et al., 2005; Partilla et al., 2006, Young and Glennon, 2008) and biological effects (Fantegrossi et al., 2003; Bondareva et al., 2005).

Drug discrimination studies with racemic MDMA suggest that its interoceptive effects are predominantly stimulant-like. For example, rats trained to discriminate the psychostimulant $S(+)$-amphetamine, but not those trained to discriminate the hallucinogen 1-(2,5-dimethoxy-4methylphenyl)-2-aminopropane (DOM), generalized their responding to racemic MDMA (Glennon et al., 1982). Conversely, rats trained to discriminate racemic MDMA from saline generalized their responding to $S(+)$-amphetamine, but not to DOM (Glennon et al., 1986; Oberlender and Nichols, 1988; Glennon, 1989). Amphetamine-like discriminative stimulus effects of racemic MDMA have also been demonstrated in pigeons (Evans and Johanson, 1986), rhesus monkeys (Kamien et al., 1986), and humans (Johanson et al., 2006). Nevertheless, some studies (e.g., Oberlender and Nichols, 1988) have failed to observe complete substitution of MDMA to an amphetamine training stimulus. Furthermore, in a three-choice drug discrimination procedure, rats have been trained to discriminate among the interoceptive effects induced by racemic MDMA, $S(+)$-amphetamine, and saline (Goodwin and Baker, 2000), which indicates that the stimulus effects of these compounds are not entirely congruent.

The optical isomers of MDMA also have been examined in drug discrimination studies, although such studies are relatively few in number. In rats, a DOM training stimulus did not generalize to either optical isomer of MDMA, but animals trained to $S(+)$-amphetamine generalized their responding to $S(+)$-MDMA but not to $R(-)$-MDMA (Glennon et al., 1982). Another study, however, reported that neither isomer of MDMA fully substituted for a $S(+)$-amphetamine stimulus (Oberlender and Nichols, 1988). In rats, both $S(+)$-MDMA and $R(-)$-MDMA substituted for a racemic MDMA stimulus (Glennon et al., 1986; Schechter, 1987; Oberlender and Nichols, 1988; Glennon, 1989), indicating some commonality of stimulus effects among the enantiomers in these species.

Human MDMA users may take more than a single dose per session, often "boosting" with subsequent pills after the interoceptive effects of the first dose begin to decrease. Repetitive administration of MDMA results in a nonlinear accumulation in the plasma of rats (Chu et al., 1996), squirrel monkeys (Mechan et al., 2006; Mueller et al., 2008), and humans (de la Torre et al., 2000), but the metabolic disposition of MDMA in the mouse is largely unknown. If the interoceptive effects of racemic MDMA are primarily mediated by one of the stereoisomers, differences in plasma accumulation or metabolism perhaps could result in unexpected toxicities (i.e., overdose) because of dose-disproportionate increases in plasma concentrations upon repeated drug administration.

The present studies were undertaken to investigate the congruence among the discriminative stimulus effects of MDMA and its enantiomers in mice and the relationship between pharmacokinetic variables and discriminative performance. Separate groups of animals were trained to discriminate $1.5 \mathrm{mg} / \mathrm{kg} S(+)-\mathrm{MDMA}$ or $R(-)$-MDMA, or their combination as $3.0 \mathrm{mg} / \mathrm{kg}$ racemic MDMA, using a two-lever procedure where injection-appropriate responding was reinforced with liquid food. The time courses of the interoceptive effects of the training doses were established, and cross sub- stitutions were made among all three compounds using a cumulative dosing procedure. In addition, pharmacokinetic variables were assessed after single injections and after cumulative dosing. To our knowledge, these studies represent the first assessments of the discriminative stimulus effects and pharmacokinetic dispositions of MDMA and its enantiomers in mice.

\section{Materials and Methods}

\section{Animals}

Behavioral Studies. Male Swiss-Webster mice (Charles River Laboratories, Inc., Wilmington, MA) initially weighing 20 to $25 \mathrm{~g}$ were housed in groups of three per $44.5-\times 22.3-\times 12.7-\mathrm{cm}$ Plexiglas cage at the Yerkes National Primate Research Center (Atlanta, GA). Lab Diet rodent chow (Laboratory Rodent Diet 5001; PMI Feeds, Inc., St. Louis, MO) and filtered water were available ad libitum in the rodent vivarium that was maintained at a temperature of $22 \pm$ $2^{\circ} \mathrm{C}$ at 45 to $50 \%$ humidity. Lights were set to a 12 -h light/dark cycle.

Pharmacokinetic Studies. Male Swiss-Webster mice (weighing between 30 and $40 \mathrm{~g}$ ) were housed in groups of three per 44.5- $\times$ 22.3- $\times 12.7-\mathrm{cm}$ plastic cage at the Institut Municipal d'Investigació Mèdica (IMIM) in Barcelona, Spain. Free access to food and water was maintained at all times while animals were housed in the vivarium, which was temperature-controlled at $21.5 \pm 1^{\circ} \mathrm{C}$. Within the animal housing room, lights were set to a 12 -h light/dark cycle.

All studies were carried out in accordance with the Declaration of Helsinki and with the Guide for the Care and Use of Laboratory Animals (Institute of Laboratory Animal Resources, 1996). Experimental protocols were approved by the Animal Care and Use Committees at the Yerkes National Primate Research Center of Emory University and IMIM.

\section{Procedure}

Behavioral Studies. Six modified operant-conditioning chambers (model ENV-008; MED Associates, St. Albans, VT) were individually enclosed in larger lightproof Malaguard sound-attenuating cubicles (model ENV-022M; MED Associates), each of which contained a house light and exhaust fan. Chambers were bisected to allow for the simultaneous testing of two animals at a time, and the stainless steel bar flooring was overlaid with chicken wire. Animals tested simultaneously in the same chamber were conspecifics within the same home cage during periods when they were not being tested and were separated from each other within the chamber by an opaque polycarbonate wall during experimental sessions. The side wall of each chamber compartment used in these studies was equipped with a spout through which liquid reinforcement was delivered, driven by an infusion pump mounted outside the chamber. The spout was centered between two retractable levers and positioned just beneath a red stimulus light, which was illuminated during reinforcer delivery. Our procedure for establishing operant performance and discriminative control by drug injections in the mouse was described previously (Yarosh et al., 2007).

Lever Training. Mice were trained daily to respond in two-lever operant conditioning boxes, reinforced by $2 \mathrm{~s}$ of access to a palatable liquid reinforcer (vanilla-flavored coffee creamer diluted 1:1 with water) from a syringe pump. Upon completion of the response requirement on either lever, that lever was retracted and reinforcement was delivered. After a brief (10 s) time-out (TO), mice were required to complete the response requirement on the remaining lever. Both levers were reintroduced into the chamber after the 10-s TO. In this manner, mice received equivalent reinforcement from each lever, and no subsequent biases for one lever or the other were noted. Animals were initially maintained on a fixed ratio (FR) 1 schedule of reinforcement in session lasting $60 \mathrm{~min}$ or until 60 reinforcers had been earned (whichever came first). The FR value increased by one increment every 20 th reinforcer earned within a 
given session, and the FR value achieved was carried over between sessions until mice were responding under an FR10. This segment of the training was complete when mice reached an FR10 and worked consistently for the reinforcer for at least 5 consecutive days.

Discrimination Training. Each group of mice $(n=6)$ was trained during daily 30 -min sessions to discriminate their respective drug [3.0 $\mathrm{mg} / \mathrm{kg}$ racemic MDMA, $1.5 \mathrm{mg} / \mathrm{kg} S(+)-M D M A$, or 1.5 $\mathrm{mg} / \mathrm{kg} R(-)$-MDMA] from saline vehicle. When animals were injected intraperitoneally with training drug, responses on the drug lever produced the reinforcer. When administered a saline injection, responses on the saline lever were reinforced. For both injection conditions, a single response on the incorrect lever resulted in retraction of that lever and extinction of the house light for a 30-s TO. During this TO, the injection-appropriate lever remained extended into the chamber, but responses on it had no programmed consequences. After the elapse of the TO, the responding under an FR10 on the remaining, injection-appropriate lever was reinforced. Injection-appropriate responding was calculated as the number of correct responses divided by the total number of responses, but given the particulars of this procedure, values were never less than $90 \%$ (unless behavior was suppressed). We have found that a more useful metric for discriminative performance during training under these conditions is percentage correct choices, which was calculated as the number of reinforcers earned divided by the total number of opportunities to make a choice between the two levers. The animals were switched from saline to drug or vice versa for the next day of training if they achieved the criteria of greater than $80 \%$ correct choices. After 3 consecutive training days where discriminative performance was above criteria, animals were tested as described below and then returned to training on the following day. If mice received the same injection type for 3 consecutive days without reaching the criteria of $80 \%$ correct choices, they were given a performance day where the mice received no injection and were reinforced on an FR10 schedule as per during lever training.

Substitution Testing. Testing to derive a dose-response curve was divided into four individual components separated by 10-min intervals to allow for the use of a cumulative dosing protocol. All such tests were conducted under extinction conditions. For the first component, test animals were administered an injection of $0.03 \mathrm{mg} / \mathrm{kg}$ for $S(+)$-MDMA and $R(-)$-MDMA or $0.1 \mathrm{mg} / \mathrm{kg}$ for racemic MDMA. After the interval, both levers were extended, and the mouse was required to complete 10 total responses on either lever to advance to the next component. If the animal did not complete 10 responses on a lever in $5 \mathrm{~min}$, the component and session were terminated. If the animal completed 10 responses on a single lever, both levers were retracted, no reinforcer was delivered, and the mouse received the next dose of test drug. The next testing component was started after the 10-min interval for a total of four components. Mice were acclimated to this procedure in two or three sessions in which saline was administered before the start of each component. The cumulative doses after each component were $0.03,0.1,0.3$, and $1.0 \mathrm{mg} / \mathrm{kg}$ for $S(+)$-MDMA and $R(-)$-MDMA and $0.1,0.3,1.0$, and $1.7 \mathrm{mg} / \mathrm{kg}$ for racemic MDMA. In this manner, each group of mice was tested for generalization to $S(+)$-MDMA, $R(-)$-MDMA, and racemic MDMA.

Time Course Testing. Animals were administered a single injection of the training dose of their respective drug and tested at 10 -min intervals after injection for $80 \mathrm{~min}$. With the exception of session duration and the administration of only a single injection, time course testing was identical to substitution testing.

\section{Pharmacokinetic Studies}

Plasma Samples. In the first set of experiments, five mice were injected intraperitoneally with $1.5 \mathrm{mg} / \mathrm{kg} R(-)-\mathrm{MDMA}, 1.5 \mathrm{mg} / \mathrm{kg}$ $S(+)-M D M A$, or $3.0 \mathrm{mg} / \mathrm{kg}$ racemic MDMA. Blood samples $(\sim 200 \mu \mathrm{l})$ were collected from the orbital sinus of each mouse at $10,30,60$, or $80 \mathrm{~min}$ in $1 \mathrm{ml}$ of heparinized polyurethane tubes (BD Biosciences, San Jose, CA) containing $3 \mu \mathrm{l}$ of $250 \mathrm{mM}$ sodium bisulfite. A total of 60 samples from 60 mice were collected; five samples for each of the four time points across three drug conditions. In a second experiment, two groups of mice were injected intraperitoneally with either a single bolus $1.7 \mathrm{mg} / \mathrm{kg}$ racemic MDMA dose or cumulative doses of racemic MDMA (first injection at $0 \mathrm{~min}, 10 \mathrm{~min}$ between each injection of doses: $0.1,0.2,0 ., 0.7 \mathrm{mg} / \mathrm{kg}$ i.p.). Blood samples were collected at $40 \mathrm{~min}$ after the single bolus injection and at 10, 20,30, and 40 min during the cumulative component in $1 \mathrm{ml}$ of heparinized polyurethane tubes (BD Biosciences) containing $3 \mu \mathrm{l}$ of $250 \mathrm{mM}$ sodium bisulfite. There were thus a total of 15 samples collected from 15 mice; three for the single bolus dose and three at each of the four time points during the cumulative dosing. All samples were immediately stored on ice, then centrifuged at $4^{\circ} \mathrm{C}$ at $3000 \mathrm{rpm}$ for $10 \mathrm{~min}$. Plasma was separated and stored at $-20^{\circ} \mathrm{C}$ for further analysis. All the procedures followed in the present work were in compliance with the European Community Council Directive (86/609/EEC).

Sample Preparation. Quantification of enantiomers was performed by gas chromatography/mass spectrometry after a chiral derivatization. Sample volumes were $50 \mu \mathrm{l}$, and final volumes of $1 \mathrm{ml}$ were obtained by dilution with blank plasma. Extraction was done as described by Pizarro et al. (2003). To reconstituted extracts (in $200 \mu \mathrm{l}$ of methanol), $4 \mathrm{ml}$ of ethyl acetate/ $\mathrm{NH}_{3}(2 \%)$ and an excess of anhydrous $\mathrm{Na}_{2} \mathrm{SO}_{4}$ was added. Tubes were stacked in an end-to-end mixer at 40 movements/min for $20 \mathrm{~min}$ and then centrifuged at $3500 \mathrm{rpm}$ for $10 \mathrm{~min}$. The solvent phase was transferred to a clean tube. This procedure was repeated twice, and the final volume of solvent was taken to dryness under nitrogen steam at $40^{\circ} \mathrm{C}, 15$ psi. Dried extracts were kept in a vacuum oven $\left(40^{\circ} \mathrm{C}\right)$ for $30 \mathrm{~min}$. Finally, derivatization was performed with minor modifications according to a procedure described previously (Pizarro et al., 2003); the amine was derivatized using $20 \mu \mathrm{l}$ of $190 \mathrm{mM} R(-)$ - $\alpha$-methoxy- $\alpha$-trifluoromethylphenylacetyl chloride in $2 \mathrm{ml}$ of ethyl acetate/hexane (50:50) that contained $0.015 \%$ triethylamine, and phenols were derivatized with $30 \mu \mathrm{l}$ of $1,1,1,3,3,3$-hexamethyldisilazane at $60^{\circ} \mathrm{C}$ for $1 \mathrm{~h}$.

Instrumentation. A gas chromatograph $(6890 \mathrm{~N}$; Agilent Technologies, Santa Clara, CA), equipped with a mass selective detector (5975; Agilent Technologies) and an autosampler injector (7683 series; Agilent Technologies), were used. Gas chromatography conditions for chiral analysis included a splitless injection mode $(0.95$ min); column, Phenomenex (Torrance, CA) cross-linked 5\% phenyl/ $95 \%$ dimethylpolysiloxane $(30-\mathrm{m} \times 0.25-\mathrm{mm} \times 0.25-\mu \mathrm{m}$ film thickness); injection port temperature, $250^{\circ} \mathrm{C}$; carrier gas, helium; flow rate, $1 \mathrm{ml} / \mathrm{min}$; temperature program, from 150 to $300^{\circ} \mathrm{C}$ at $20^{\circ} \mathrm{C} /$ min; initial time, $1.10 \mathrm{~min}$; final time, $3 \mathrm{~min}$; and injection volume, 3 $\mu l$. Mass spectrometry conditions were: selected ion monitoring mode; positive chemical ionization, $240 \mathrm{eV}$; and ion source temperature, $300^{\circ} \mathrm{C}$. The reagent gas used was methane. Mass $/$ charge $(\mathrm{m} / z)$ values selected for quantification of analytes were as follows: 410 for MDMA- $N$-MTP, 396 for MDA- $N$-MTP, 542 for HHMA- $N$-MTP- $O$-bisTMS, 484 for HMMA- $N$-MTP-O-TMS, 528 for HHA- $N$-MTP-O-bisTMS, and 470 for HMA- $N$-MTP-O-TMS. Mass/charge $(\mathrm{m} / z)$ values for internal standards were: 415 for MDMA-D5- $N$-MTP, 401 for MDA-D5- $N$-MTP, 454 for pholedrine- $N$-MTP-O-TMS, and 500 for DHBA- $N$-MTP-O-bis-TMS. For identification and confirmation of the conversion from one MDMA enantiomer to the other, samples were injected using the same chromatographic conditions, with $\mathrm{m} / \mathrm{z}$ values selected for MDMA-N-MTP as 410, 378, and 163.

Drugs. All chemicals (analysis or reagent grade) were obtained from Lipomed AG (Arlesheim, Switzerland), Radian Research, Inc. (Lafayette, IN), Aldrich Chemical Co. (Milwaukee, WI), Merck (Whitehouse Station, NJ), Scharlab Chemie (Barcelona, Spain), Sigma-Aldrich (St. Louis, MO), and Varian, Inc. (Palo Alto, CA). Pholedrine (4-hydroxymethamphetamine) was generously given by the Deutsche Sporthochschule, Biochemistry Department (Cologne, Germany). MDMA and its enantiomers were obtained from the National Institute on Drug Abuse Drug Supply Program.

Data Analysis. Drug discrimination data are presented as percent drug-appropriate responding (number of responses on the drugappropriate lever as a percentage of the total number of responses) 
and response rate (expressed as responses per second). The criterion for generalization was set as $80 \%$ drug-appropriate responding. Pharmacokinetic noncompartmental analyses (estimation of $C_{\max }$, $t_{\text {max }}, \mathrm{AUC}_{10-80 \min }, K_{\mathrm{e}}$ ) were performed using Microsoft Excel (PK Functions for Microsoft Excel, http://www.boomer.org/pkin/ soft.html). Pharmacokinetic parameters were estimated separately for each mouse, and the mean and S.D. of these estimations were calculated. Statistical analysis was performed using SPSS for Windows version 12.0 (SPSS Inc., Chicago, IL). Where the same groups of mice were given the same dose regimen, pharmacokinetic parameters of $R(-)$-MDMA and metabolites were compared with their equivalent $S(+)$-enantiomer using both a paired Student's $t$ test and, assuming non-normal distribution because of low sample number, the Wilcoxon nonparametric test for paired samples. Independent Student's $t$ tests and nonparametric Mann-Whitney tests were also performed to compare $R(-)$-MDMA and metabolite pharmacokinetics with their equivalent $S(+)$-enantiomer parameters from the first set of experiments [1.5 mg/kg $R(-)$-MDMA versus $1.5 \mathrm{mg} / \mathrm{kg} S(+)$ MDMA and $1.7 \mathrm{mg} / \mathrm{kg}$ racemic MDMA versus cumulative dose]. Values of $p<0.05$ were considered statistically significant.

\section{Results}

Behavioral Studies. Racemic MDMA dose-dependently and fully substituted for its training stimulus (Fig. 1A, ) at doses of 1.0 and $1.7 \mathrm{mg} / \mathrm{kg}$ and reduced response rates (Table 1). The $\mathrm{ED}_{50}$ for racemic MDMA in mice trained to discriminate $3.0 \mathrm{mg} / \mathrm{kg}$ racemic MDMA from saline was approximately $0.4 \pm 0.2 \mathrm{mg} / \mathrm{kg}$. Likewise, $S(+)$-MDMA dose-dependently and fully substituted for the racemic MDMA training cue (Fig. 1A, $\mathbf{\Lambda}$ ) at a dose of $1.0 \mathrm{mg} / \mathrm{kg}$ and reduced response rates (Table 1). The $\mathrm{ED}_{50}$ for $S(+)$-MDMA in mice trained to discriminate $3.0 \mathrm{mg} / \mathrm{kg}$ racemic MDMA from saline was approximately $0.17 \pm 0.1 \mathrm{mg} / \mathrm{kg}$. It is interesting that $R(-)$ MDMA did not substitute for the racemic MDMA training cue at any dose (Fig. $1 \mathrm{~A}, \nabla) . R(-)$-MDMA engendered only saline-appropriate responding up to doses that completely suppressed responding in three animals (Table 1), precluding an $\mathrm{ED}_{50}$ determination for this compound in these animals.

$S(+)$-MDMA dose-dependently and fully substituted for its training stimulus (Fig. 1B, $\mathbf{\Delta}$ ) at a dose of $1.0 \mathrm{mg} / \mathrm{kg}$ and reduced response rates (Table 2 ). The $\mathrm{ED}_{50}$ for $S(+)$-MDMA in mice trained to discriminate $1.5 \mathrm{mg} / \mathrm{kg} S(+)$-MDMA from saline was approximately $0.3 \pm 0.2 \mathrm{mg} / \mathrm{kg}$. Racemic MDMA also dose-dependently and fully substituted for the $S(+)$ -
MDMA training cue (Fig. 1B, ) at doses of 0.3 and $1.0 \mathrm{mg} / \mathrm{kg}$ and reduced response rates (Table 2). The $\mathrm{ED}_{50}$ for racemic MDMA in mice trained to discriminate $1.5 \mathrm{mg} / \mathrm{kg} S(+)$ MDMA from saline was approximately $0.1 \pm 0.1 \mathrm{mg} / \mathrm{kg}$. In contrast to the results obtained in mice trained to discriminate racemic MDMA from saline, $R(-)$-MDMA substituted dose-dependently for the $S(+)$-MDMA training cue, and mice fully generalized their responding at $1.0 \mathrm{mg} / \mathrm{kg}$ (Fig. 1B, $\nabla$ ). The $\mathrm{ED}_{50}$ for $R(-)$-MDMA in mice trained to discriminate 1.5 $\mathrm{mg} / \mathrm{kg} S(+)$-MDMA from saline was approximately $0.17 \pm$ $0.1 \mathrm{mg} / \mathrm{kg}$. As with the other compounds tested, $R(-)$-MDMA dose-dependently suppressed response rates to some extent (Table 2).

$R(-)$-MDMA dose-dependently and fully substituted for its training stimulus (Fig. $1 \mathrm{C}, \nabla$ ) at a dose of $1.0 \mathrm{mg} / \mathrm{kg}$ and reduced response rates (Table 3 ). The $\mathrm{ED}_{50}$ for $R(-)$-MDMA in mice trained to discriminate $1.5 \mathrm{mg} / \mathrm{kg} R(-)$-MDMA from saline was approximately $0.17 \pm 0.1 \mathrm{mg} / \mathrm{kg}$. $S(+)$-MDMA potently substituted for the $R(-)$-MDMA training cue, reaching full substitution at doses of 0.3 and $1.0 \mathrm{mg} / \mathrm{kg}$ (Fig. 1C, $\mathbf{A}$ ) and reduced response rates (Table 3 ). The $\mathrm{ED}_{50}$ for $S(+)$ MDMA in mice trained to discriminate $1.5 \mathrm{mg} / \mathrm{kg} R(-)$ MDMA from saline was approximately $0.03 \pm 0.1 \mathrm{mg} / \mathrm{kg}$. In an example of nonsymmetrical generalization, racemic MDMA dose-dependently and fully substituted for the $R(-)$ MDMA training cue (Fig. 1C, ) at a dose of $0.3 \mathrm{mg} / \mathrm{kg}$ and reduced response rates (Table 3 ). The $\mathrm{ED}_{50}$ for racemic MDMA in mice trained to discriminate $1.5 \mathrm{mg} / \mathrm{kg} R(-)$ MDMA from saline was approximately $0.056 \pm 0.1 \mathrm{mg} / \mathrm{kg}$.

Assessment of the time courses of the discriminative stimulus effects engendered by the training doses of MDMA and its enantiomers revealed interesting differences between compounds. Almost complete drug-appropriate responding was engendered by $3.0 \mathrm{mg} / \mathrm{kg}$ racemic MDMA and $1.5 \mathrm{mg} / \mathrm{kg}$ $S(+)$-MDMA at time points from 10 to $60 \mathrm{~min}$ after injection, and responses were incrementally reallocated to the salineappropriate lever at later time points for both drugs (Fig. 2, - and triangles). In contrast, mice injected with $1.5 \mathrm{mg} / \mathrm{kg}$ $R(-)$-MDMA evenly distributed their responses on both levers 10 min after injection, allocated more responses to the drug-appropriate lever at 20 and 30 min after injection, then gradually shifted their behavior back to the saline-appropriate lever for the duration of the test (Fig. 2, $\nabla$ ).

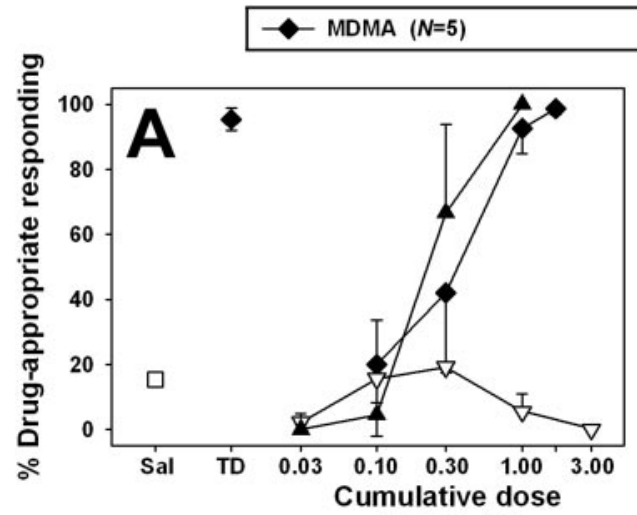

( $\mathrm{mg} / \mathrm{kg}$, ip)

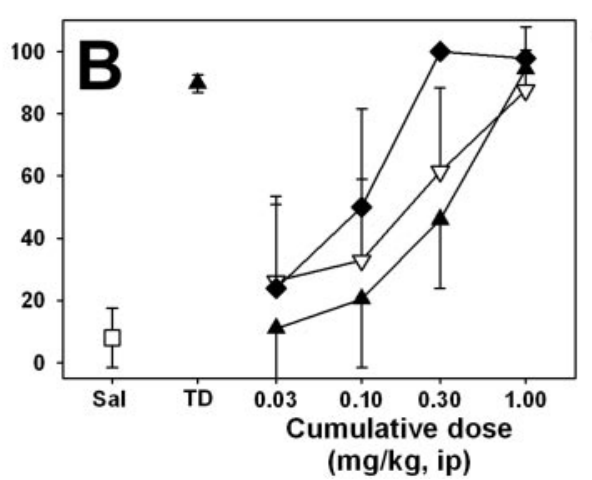

(mg/kg, ip)

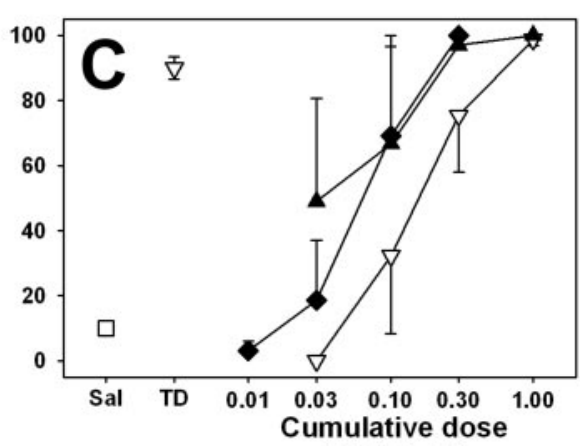

( $\mathrm{mg} / \mathrm{kg}$, ip)

Fig. 1. Discriminative stimulus effects of MDMA $(\diamond), S(+)$-MDMA $(\boldsymbol{\Delta})$, and $R(-)$-MDMA (open inverted triangles) in mice trained to discriminate 3.0 $\mathrm{mg} / \mathrm{kg}$ MDMA (A), $1.5 \mathrm{mg} / \mathrm{kg} S(+)$-MDMA (B), or $1.5 \mathrm{mg} / \mathrm{kg} R(-)$-MDMA (C) from saline. Each point represents the mean of all animals per group; error bars, \pm S.E.M. Abscissae, dose of drug expressed as milligrams per kilogram and plotted on a log scale. The points at Sal and TD represent saline and drug training sessions. Ordinates, percentage drug-appropriate responding. 


\section{TABLE 1}

Rates of responding (per second) after injection with various drugs and doses in mice trained to discriminate $3.0 \mathrm{mg} / \mathrm{kg}$ racemic MDMA from saline

\begin{tabular}{lccc}
\hline \multicolumn{1}{c}{ Dose } & MDMA & $S(+)$-MDMA & $R(-)$-MDMA \\
\hline Saline & $0.45 \pm 0.13^{a}$ & & \\
0.03 & & $0.44 \pm 0.11$ & $0.54 \pm 0.06$ \\
0.1 & $0.61 \pm 0.22$ & $0.91 \pm 0.43$ & $0.20 \pm 0.10$ \\
0.3 & $0.35 \pm 0.17$ & $0.56 \pm 0.34$ & $0.25 \pm 0.14$ \\
1.0 & $0.23 \pm 0.07$ & $0.28 \pm 0.12$ & $0.26 \pm 0.18$ \\
1.7 & $0.19 \pm 0.16$ & & \\
3.0 & $0.30 \pm 0.14^{*}$ & & $0.07 \pm 0.06$ \\
\hline
\end{tabular}

${ }^{a}$ Response rates obtained in training sessions initiated by a single injection of either saline or the racemic MDMA training dose; all other rates were obtained in test sessions using cumulative dosing.

TABLE 2

Rates of responding (per second) after injection with various drugs and doses in mice trained to discriminate $1.5 \mathrm{mg} / \mathrm{kg} S(+)$-MDMA from saline

\begin{tabular}{lcll}
\hline \multicolumn{1}{c}{ Dose } & MDMA & $S(+)$-MDMA & $R(-)$-MDMA \\
\hline Saline & & $0.91 \pm 0.26^{a}$ & \\
0.03 & $0.55 \pm 0.13$ & $0.54 \pm 0.37$ & $0.49 \pm 0.15$ \\
0.1 & $0.43 \pm 0.13$ & $0.32 \pm 0.19$ & $0.48 \pm 0.17$ \\
0.3 & $0.31 \pm 0.16$ & $0.33 \pm 0.14$ & $0.35 \pm 0.17$ \\
1.0 & $0.21 \pm 0.15$ & $0.19 \pm 0.09$ & $0.32 \pm 0.24$ \\
1.5 & & $0.22 \pm 0.06^{a}$ & \\
\hline
\end{tabular}

${ }^{a}$ Response rates obtained in training sessions initiated by a single injection of either saline or the $S(+)$-MDMA training dose; all other rates were obtained in test sessions using cumulative dosing.

\section{TABLE 3}

Rates of responding (per second) after injection with various drugs and doses in mice trained to discriminate $1.5 \mathrm{mg} / \mathrm{kg} R(-)$-MDMA from saline

\begin{tabular}{lccc}
\hline \multicolumn{1}{c}{ Dose } & MDMA & $S(+)$-MDMA & $R(-)$-MDMA \\
\hline Saline & & & $0.68 \pm 0.23^{a}$ \\
0.01 & $0.41 \pm 0.08$ & & \\
0.03 & $0.33 \pm 0.20$ & $0.72 \pm 0.33$ & $0.43 \pm 0.19$ \\
0.1 & $0.55 \pm 0.12$ & $0.29 \pm 0.22$ & $0.49 \pm 0.39$ \\
0.3 & $0.27 \pm 0.04$ & $0.34 \pm 0.23$ & $0.39 \pm 0.26$ \\
1.0 & & $0.14 \pm 0.09$ & $0.33 \pm 0.25$ \\
1.5 & & & $0.43 \pm 0.21^{a}$ \\
\hline
\end{tabular}

${ }^{a}$ Response rates obtained in training sessions initiated by a single injection of either saline or the $R(-)$-MDMA training dose; all other rates were obtained in test sessions using cumulative dosing.

Pharmacokinetic Studies. Administration of a single bolus dose of $3.0 \mathrm{mg} / \mathrm{kg}$ racemic MDMA (Table 4) resulted in a higher accumulation of $S(+)$-MDMA than of $R(-)$-MDMA. $\operatorname{Both}_{\mathrm{AUC}_{0-80 \min }}(p=0.0431, z=-2.023 ; p=0.0138, t=$ $-4.183)$ and $C_{\max }(p=0.0431, z=-2.023)$ were significantly different among the enantiomers (Table 4). $S(+)$ - and $R(-)$ MDMA were found at higher concentrations than any metabolites of racemic MDMA. In the next steps of MDMA metabolic disposition, more differences are seen among metabolites plasma concentrations depending on the enantiomer considered. For MDA, the $N$-demethylation metabolic product of MDMA, $\mathrm{AUC}_{0-80}$ min for $S(+)-\mathrm{MDA}$ was almost 5 times higher than $R(-)$-MDA ( $p=0.0431, z=-2.023, p=0.0004$, $t=-11.160$ ), and the $C_{\max }$ for $S(+)$-MDA was almost 3 times higher than $R(-)$-MDA ( $p=0.0431, z=-2.023, p=0.0080$, $t=-4.912$ ) (Table 4). Although no significant differences were noted among metabolites resulting from the $O$-demethylation and further $O$-methylation of MDMA and MDA (HMMA, HMA, or HHA), it seems that metabolites derived from the $S(+)$-isomers are more efficiently formed because concentrations of $R(-)$-HHA were below the limit of detec-

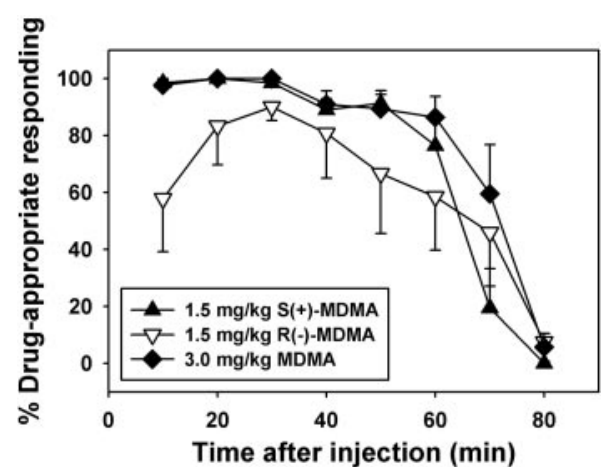

Fig. 2. Time course of the discriminative stimulus effects of training doses of $3.0 \mathrm{mg} / \mathrm{kg}$ MDMA ( ), $1.5 \mathrm{mg} / \mathrm{kg} S(+)-M D M A(\Delta)$, and $1.5 \mathrm{mg} / \mathrm{kg}$ $R(-)$-MDMA $(\nabla)$ in mice trained to discriminate these injections from saline. Each point represents the mean of all animals per group; error bars, \pm S.E.M. Abscissa, time after injection, in minutes. Ordinate, percentage drug-appropriate responding.

tion. A similar metabolic pattern is seen after the administration of a single bolus dose of $1.5 \mathrm{mg} / \mathrm{kg} R(-)$-MDMA (Table 5) and $S(+)$-MDMA (Table 6). The observed $\mathrm{AUC}_{0-80 \text { min }}$ for $R(-)$-MDA after injection of $R(-)$-MDMA was more than 4-fold lower than was the $\mathrm{AUC}_{0-80}$ min for $S(+)$-MDA after injection of $S(+)$-MDMA $(p=0.0079, z=-2.611, p=0.0017$, $t=-4.647)$. Likewise, the $C_{\max }$ for $R(-)$-MDA after injection of $R(-)$-MDMA was approximately 4 -fold lower than was the $C_{\max }$ for $S(+)$-MDA after injection of $S(+)$-MDMA ( $p=$ $0.0079, z=-2.611, p=0.0014, t=-4.784$ ). No other pharmacokinetic parameters were significantly different across administration of the two MDMA enantiomers. It is worthwhile to note that as for the racemate experiment, the catechol metabolite HHA is only detected after the administration of $S(+)$-MDMA (single bolus).

Plasma concentrations of the enantiomers of MDMA and the enantiomers of the MDMA metabolites MDA, HMMA, HHMA, and HHA were quantified after a cumulative racemic MDMA dosing procedure identical to that used in the drug discrimination studies or after a single bolus dose of 1.7 $\mathrm{mg} / \mathrm{kg}$ racemic MDMA. Accumulation of the MDMA metabolites was time dependent (Table 7). At the 10-min time point (after a dose of $0.1 \mathrm{mg} / \mathrm{kg}$ racemic MDMA) and at all other time points, there were no significant differences between the concentrations of the MDMA enantiomers. At the 20-min time point (a cumulative dose of $0.3 \mathrm{mg} / \mathrm{kg}$ racemic MDMA), concentrations of the MDMA enantiomers doubled, and $S(+)$ MDA was quantified in a single sample, whereas $R(-)$-MDA concentrations were below the level of detection in all samples analyzed. A similar pharmacokinetic profile was observed at the 30-min time point (a cumulative dose of 1.0 $\mathrm{mg} / \mathrm{kg}$ racemic MDMA). Concentrations of the MDMA enantiomers were increased approximately 4 -fold over the initial sample, concentrations of $S(+)$-MDA were increased more than 10 -fold over the previous sample, and $R(-)$-MDA remained below the limit of detection. A significantly broader metabolite profile was quantified at the 40 -min time point (a cumulative dose of $1.7 \mathrm{mg} / \mathrm{kg}$ racemic MDMA). Concentrations of the MDMA enantiomers were increased approximately 5 -fold over the initial sample, and concentrations of $S(+)$-MDA were stable (in comparison with the previous sample), whereas $R(-)$-MDA accumulation reached the limit of detection, and its concentration was 3-fold less than that of $S(+)$-MDA. In addition, HMMA enantiomers were detected, 
in addition to those of HHMA and HHA. There were no differences in the concentrations of these MDMA metabolites. It is important that concentrations of both $S(+)$-MDMA and $R(-)$-MDMA were lower after a single bolus dose of 1.7 $\mathrm{mg} / \mathrm{kg}$ racemic MDMA compared with an equivalent cumulative dose spread over four administrations (Table 7; Fig. 3), mimicking the manner in which drug discrimination tests were performed. Concentrations of the enantiomers of the MDMA metabolites MDA, HMMA, HHMA, and HHA were higher after administration of a single bolus dose of 1.7 $\mathrm{mg} / \mathrm{kg}$ racemic MDMA compared with the concentrations observed using the cumulative dosing procedure, although the relatively small sample size precluded the detection of statistical significance. The sum of recovered concentrations of metabolites after the single bolus administration compensates differences seen in MDMA concentrations comparing both administration protocols.

Evidence for the interconversion of the MDMA enantiomers after administration of a $1.5 \mathrm{mg} / \mathrm{kg}$ concentration of either isomer is shown in Fig. 4. The parent ion of MDMAN-MTP $(\mathrm{m} / \mathrm{z})$ plus two ions from the fragmentation of the same are found at the retention times of $R(-)$-MDMA and $S(+)$-MDMA. Upon calculating the concentrations after separate doses of $1.5 \mathrm{mg} / \mathrm{kg} R(-)-M D M A$ and $S(+)-M D M A$, it was found that $3.5 \%$ of $R(-)$-MDMA is converted to $S(+)$ MDMA, whereas $9.9 \%$ of $S(+)$-MDMA is converted to $R(-)$ MDMA. This interconversion of the MDMA enantiomers has not been demonstrated previously in vivo.

\section{Discussion}

MDMA and its enantiomers were successfully trained as discriminative stimuli in mice, but, consistent with a previous report in the rat (Bondareva et al., 2005), $R(-)$-MDMA was notably more difficult to train than racemic MDMA or the $S(+)$-enantiomer. Use of a higher $R(-)$-MDMA training dose might have accelerated acquisition of the discrimination, but the experimental design for these studies necessitated that $S(+)$ - and $R(-)$-MDMA be trained at the same dose, half the training dose used for racemic MDMA, to allow for a fair assessment of cross-generalization among these compounds during substitution tests. Thus, $R(-)$-MDMA may simply be less potent than $S(+)$ - or racemic MDMA in terms of discriminative stimulus effects. Likewise, the lethal effects of $R(-)$-MDMA are less potent than those of $S(+)$ - and racemic MDMA in mice (Fantegrossi et al., 2003), and $R(-)$ MDMA is 4-fold less potent than $S(+)$ - and racemic MDMA in suppressing operant responding in the mouse (Glennon et al., 1987). Such potency differences are not universally reported, however, because head twitch behavior elicited by $S(+)$ - and $R(-)$-MDMA occurs over a comparable dose range (Fantegrossi et al., 2005).

$S(+)$ - and racemic MDMA elicit a similar cluster of behavioral and physiological effects in the mouse, including locomotor stimulation and hyperthermia (Fantegrossi et al., 2003; Herin et al., 2005). Thus, the reciprocal generalization here reported between $S(+)$ - and racemic MDMA is consistent with these and other in vivo comparisons of these drugs. Likewise, both enantiomers of MDMA elicit head twitch behavior in the mouse, albeit via different mechanisms (Fantegrossi et al., 2005), so it was not entirely unexpected that both $S(+)$ - and $R(-)$-MDMA would substitute for each other. 
TABLE 5

Pharmacokinetic parameters of $R(-)$-MDMA and its respective metabolites after a $1.5 \mathrm{mg} / \mathrm{kg}$ dose in mice (values for $t_{\text {max }}$ are given as a median)

\begin{tabular}{|c|c|c|c|c|c|}
\hline & $R(-)$-MDMA & $R(-)$-MDA & $R(-)$-HMMA & $R(-)$-HMA & $R(-)-\mathrm{HHMA}^{a}$ \\
\hline $\operatorname{AUC}_{0-80}(\mu \mathrm{g} / \mathrm{min} / \mathrm{l})$ & $9821.8 \pm 4275.8$ & $549.4 \pm 427.9^{*}$ & $527.3 \pm 34.4$ & $436.7 \pm 216.5$ & 230.0 \\
\hline$C_{\max }(\mu \mathrm{g} / \mathrm{l})$ & $310.1 \pm 179.2$ & $12.0 \pm 7.3^{*}$ & $10.7 \pm 1.7$ & $8.7 \pm 3.0$ & 14.2 \\
\hline$t_{\max }(\min )$ & $10 \pm 8.9$ & $30 \pm 28.6$ & $45 \pm 17.3$ & $45 \pm 24.5$ & 10 \\
\hline$K_{\mathrm{e}}\left(\min ^{-1}\right)$ & $0.031 \pm 0.011$ & & & & \\
\hline
\end{tabular}

\footnotetext{
${ }^{a}$ Observations from a single sample.

* Significant differences between $R(-)$-enantiomer after administration of $1.5 \mathrm{mg} / \mathrm{kg} R(-)$-MDMA and $S(+)$-enantiomer after administration of $1.5 \mathrm{mg} / \mathrm{kg} S(+)$-MDMA $(p<0.05, n=5)$.
}

TABLE 6

Pharmacokinetic parameters of $S(+)$-MDMA and its respective metabolites after a $1.5 \mathrm{mg} / \mathrm{kg}$ dose in mice (values for $t_{\max }$ are given as a median) Table properties are as described in Table 2.

\begin{tabular}{|c|c|c|c|c|c|c|}
\hline & $S(+)$-MDMA & $S(+)-\mathrm{MDA}$ & $S(+)$-HMMA & $S(+)$-HMA & $S(+)-\mathrm{HHMA}^{\dagger}$ & $S(+)$-HHA \\
\hline $\operatorname{AUC}_{0-80}\left(\mu \mathrm{g} / \mathrm{min} / /^{-1}\right)$ & $14,080 \pm 3484.9$ & $2682.3 \pm 933$ & $1016.6 \pm 378.8$ & $1483.9 \pm 441.3$ & 58.8 & $2223.8 \pm 1856.6$ \\
\hline$C_{\max }(\mu \mathrm{g} / \mathrm{l})$ & $331.3 \pm 81$ & $52.2 \pm 17.3$ & $18.8 \pm 7.4$ & $92.3 \pm 9.9$ & 5.9 & $69.7 \pm 50.8$ \\
\hline$t_{\max }^{\max }(\min )$ & $30 \pm 11$ & $60 \pm 16.4$ & $60 \pm 15$ & $20 \pm 14.1$ & 80 & $80 \pm 0.1$ \\
\hline$K_{\mathrm{e}}\left(\min ^{-1}\right)$ & $0.023 \pm 0.001$ & & & & & \\
\hline
\end{tabular}

Mice trained with racemic MDMA failed to generalize their responding to substitution doses of $R(-)$-MDMA, although racemic MDMA fully substituted in mice trained with $R(-)$ MDMA. This nonreciprocal generalization might be due to perceptual masking (Wegel and Lane, 1924), in which a complex of stimulus effects may not be congruent with the individual components of that stimulus. Mice discriminating racemic MDMA from saline were, in essence, trained to a composite stimulus consisting of $S(+)$ - and $R(-)$-MDMA. In these subjects, it would seem that the stimulus effects of $S(+)$-MDMA overshadow those of $R(-)$-MDMA, such that animals trained to the racemic mixture recognize only the cue induced by $S(+)$-MDMA. The role of perceptual masking in drug discrimination has been discussed recently (Koek et al., 2006; Li et al., 2008). Alternatively, although administered at a constant dose, mice acquired the discrimination between $R(-)$-MDMA and saline more slowly than did mice trained with $S(+)$-MDMA, perhaps indicating a more subtle interoceptive effect. Thus, the failure of $R(-)$-MDMA to substitute for racemic MDMA could indicate that the discriminative stimulus effects of $R(-)$-MDMA are not as salient as are those of the racemate or the $S(+)$-enantiomer.

In studies designed to assess the time course of these discriminative stimulus effects, important differences in both onset and duration of action were uncovered among these drugs. For both racemic and $S(+)$-MDMA, full discriminative control was observed within 10 min after injection and remained largely unchanged over approximately the next 50 $\mathrm{min}$. These findings suggest that the interoceptive effects of racemic and $S(+)$-MDMA have a relatively rapid onset and last at least $60 \mathrm{~min}$ after injection. With regard to $R(-)$ MDMA, discriminative performance no greater than chance was observed 10 min after injection. Stimulus control improved over the next $20 \mathrm{~min}$, then worsened at all subsequent time points. Thus, in comparison with racemic MDMA and the $S(+)$-enantiomer, the interoceptive effects of $R(-)$ MDMA are slower in onset and shorter in duration, which might explain the difficulty we and others have encountered training $R(-)$-MDMA as a discriminative stimulus.

Pharmacokinetics also may be relevant to the discussion of the discriminative stimulus effects of $R(-)$-MDMA. The $t_{\max }$ of $R(-)$-MDMA was $10 \mathrm{~min}$, a time at which discriminative performance was no better than chance. However, the $t_{\max }$ of
$R(-)$-MDMA's main metabolite $R(-)$-MDA occurred at 30 min, coinciding with the peak discriminative stimulus effects of $R(-)$-MDMA. The $t_{\max }$ of $S(+)$-MDMA was also $10 \mathrm{~min}$, although discriminative performance after $S(+)$-MDMA injection was maximal between 10 and $30 \mathrm{~min}$. Taken together, these observations suggest that the metabolite $R(-)$-MDA (rather than its parent compound) may be responsible for the discriminative stimulus effects engendered by an injection of $R(-)$-MDMA, whereas both $S(+)$-MDMA and its metabolite $S(+)$-MDA may account for discriminative stimulus effects after $S(+)$-MDMA injection.

After a dose of racemic MDMA, the principle pathway of oxidative metabolism in Swiss-Webster mice is generally $\mathrm{N}$ demethylation to MDA, which is then $O$-demethylated to HHA followed by further methylation to HMA (Ortuño et al., 1999). Concentrations of $R(-)$-MDA were approximately $5 \%$ of those of $R(-)$-MDMA, and concentrations of $S(+)$-MDA were approximately $20 \%$ of $S(+)$-MDMA. Similar results have been observed previously (Fitzgerald et al., 1989). Given the enantioselective metabolism of MDMA after racemate administration, with $S(+)$-MDA accumulation higher than that of $R(-)$-MDA, discriminative performance after racemate administration may be due to $S(+)$-MDMA and $S(+)$ MDA. A practically complete overlap of graphs representing drug response versus time after racemic MDMA and $S(+)$ MDMA administration seems to confirm this hypothesis. Unlike mice, MDMA's main metabolic pathway in humans is $O$-demethylation to HHMA with further methylation to HMMA (Pizarro et al., 2003). This could partially explain the interspecies differences in the neuropharmacological effects of MDMA with MDA being more abundant in mouse plasma and thereby proportionately more responsible for eliciting discriminative stimulus effects in mice.

$R(-)$-MDMA was cleared preferentially to $S(+)$-MDMA after both single- and cumulative dose administration of racemic MDMA. There were differences found in MDMA's enantiomeric ratio after $3.0 \mathrm{mg} / \mathrm{kg}$ was administered; however, plasma concentrations of both MDMA enantiomers after $1.7 \mathrm{mg} / \mathrm{kg}$ were not different, agreeing with previous reports on their urinary recoveries (Lim et al., 1993). The equal concentration of MDMA enantiomers despite this enantioselective metabolism may be explained in part by an in vivo biotransformation between enantiomers. In fact, analy- 


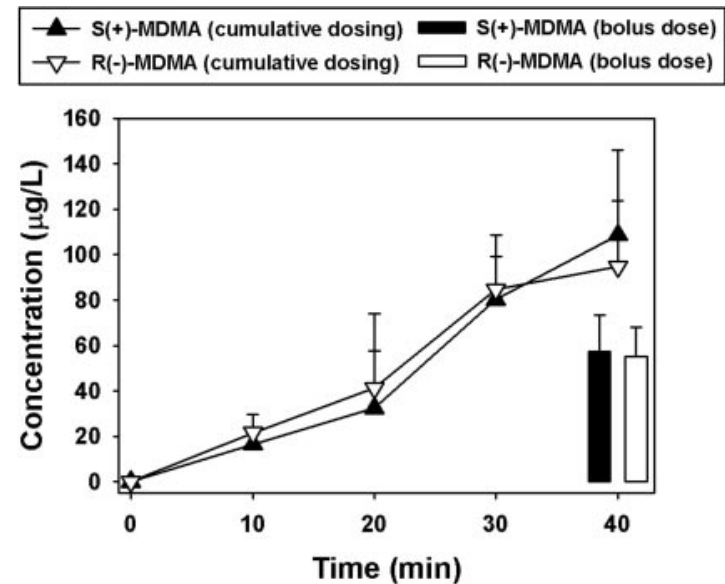

Fig. 3. $R(-)$-MDMA and $S(+)$-MDMA plasma concentrations (micrograms per liter) after a cumulative dose regimen of racemic MDMA or a single bolus dose.

sis of enantiomers of MDMA and its metabolites after pure MDMA enantiomer administration shows a 3.5 and $9.9 \%$ conversion of one enantiomer to the other after administration of $R(-)$ - and $S(+)$-MDMA, respectively. This is the first evidence of interconversion of MDMA enantiomers in vivo, although it has been reported for other chiral compounds, such as ibuprofen (Agranat and Caner, 1999) and thalidomide (Reist et al., 1998). It is difficult to extrapolate MDMA enantiomeric interconversion to humans because, to our knowledge, there are no pharmacokinetic experiments of pure MDMA enantiomer administration in man.

Cumulative doses of racemic MDMA lead to higher concentrations of MDMA compared with administration of an equivalent single dose, revealing that metabolism is decreased when the dose is administered cumulatively. Furthermore, MDA concentrations are lower after the cumulative dose compared with the single dose, suggesting autoinhibition of MDMA metabolism in mice, as also observed in humans (de la Torre et al., 2000). The inhibition of CYP2D6 is thought to cause this nonlinear pharmacokinetics in humans. Although mice do not possess CYP2D6, they express many homologous CYP2D isozymes, one or more of which may be inhibited by MDMA. Although no statistically significant differences were found because of small sample size, both cumulative and single doses of MDMA yield $R(-) / S(+)$ ratios $<1$ in all cases except for HMMA, where the ratio is $>1$ (observation based on a single sample). Metabolite concentrations in all cases are higher after a single dose compared with an equivalent cumulative dose, and $R(-) / S(+)$ ratios are similar for both single and cumulative doses. These data show that, although more MDMA is being metabolized when it is administered in a single dose, enantioselective metabolism is not affected by the pattern of dose administration, and the hypothetic enzyme inhibited after MDMA administration is not responsible for the stereochemical differences in its metabolism.

In summary, the present data confirm that all three forms of MDMA can be trained as discriminative stimuli in the mouse and that, with the exception of $R(-)$-MDMA in mice trained to discriminate the racemate, all compounds substituted for one another. As in other behavioral measures, the onset of interoceptive effects for $S(+)$-MDMA and racemic MDMA were similar to one another and were faster than for $R(-)$-MDMA. Likewise, the duration of discriminative stim- 

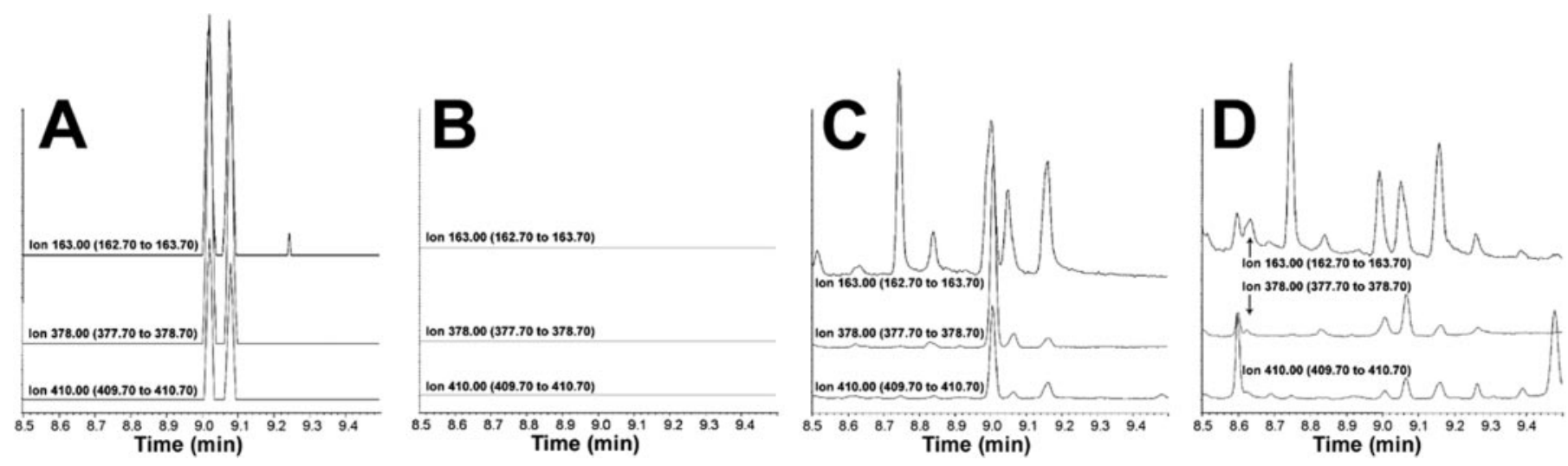

Fig. 4. Gas chromatography/chemical ionization-mass spectrometry chromatograms of $\mathrm{m} / z=410$, 378, and 163 corresponding to $R$ (-)-MDMA-N-MTP (retention time $=9.00 \mathrm{~min}$ ) and $S(+)$-MDMA-N-MTP (retention time $=9.06 \mathrm{~min}$ ) parent ions and fragmentation patterns. A, blank plasma sample spiked with $100 \mathrm{ng}$ of racemic MDMA. B, blank plasma sample. C, conversion of $R(-)$-MDMA to $S(+)$-MDMA in mouse plasma after administration of $1.5 \mathrm{mg} / \mathrm{kg} R(-)$-MDMA. D, conversion of $S(+)$-MDMA to $R(-)$-MDMA in mouse plasma after administration of $1.5 \mathrm{mg} / \mathrm{kg} S(+)$-MDMA.

ulus effects was similar for $S(+)$-MDMA and the racemate and was shortest for $R(-)$-MDMA. After a single injection of racemic MDMA, $S(+)$-MDMA and its metabolites were found in higher concentrations than $R(-)$-MDMA and its metabolites, whereas cumulative doses of MDMA lead to higher plasma concentrations compared with an equivalent bolus dose. In contrast to that observed in humans, the $\mathrm{N}$-dealkylation pathway is favored in mouse plasma with MDA as the main metabolite formed. MDA concentrations are lower after the cumulative dose compared with the single dose, which, coupled with the nonlinearity observed in MDMA pharmacokinetics after increased doses of racemic MDMA, suggests autoinhibition (or saturation) of MDMA metabolism in mice. In total, these studies suggest that the discriminative stimulus effects of racemic MDMA perhaps are driven by accumulation of $S(+)$-MDMA and $S(+)$-MDA in the mouse. As recreational use of MDMA continues, further study likely will be necessary to understand the complex behavioral and pharmacokinetic actions of this drug.

\section{Acknowledgments}

We thank Dr. Asunción Marín (Serveis de Suport a la Recerca, Universitat de Barcelona, Spain). Travel support for N.M. was generously provided by the Emory University International Research Experience for Science Students program. Expert animal husbandry services were provided by the animal care staff at Yerkes and IMIM.

\section{References}

Agranat II and Caner H (1999) Intellectual property and chirality of drugs. Drug Discov Today 7:313-321.

Bondareva T, Wesołowska A, Dukat M, Lee M, Young R, and Glennon RA (2005) $S(+)$ - and $R(-) N$-methyl-1-(3,4-methylenedioxyphenyl)-2-aminopropane (MDMA) as discriminative stimuli: effect of cocaine. Pharmacol Biochem Behav 82:531-538.

Chu T, Kumagai Y, DiStefano EW, and Cho AK (1996) Disposition of methylenedioxymethamphetamine and three metabolites in the brains of different rat strains and their possible roles in acute serotonin depletion. Biochem Pharmacol 51:789-796.

Cole JC, Bailey M, Sumnall HR, Wagstaff GF, and King LA (2002) The content of ecstasy tablets: implications for the study of their long-term effects. Addiction 97:1531-1536.

de la Torre R, Farré M, Ortuño J, Mas M, Brenneisen R, Roset PN, Segura J, and Camí J (2000) Non-linear pharmacokinetics of MDMA ("ecstasy") in humans. Br J Clin Pharmacol 49:104-109.

Evans SM and Johanson CE (1986) Discriminative stimulus properties of ( \pm )-3,4methylenedioxymethamphetamine and $( \pm)-3,4$-methylenedioxyamphetamine in pigeons. Drug Alcohol Depend 18:159-164.

Fantegrossi WE, Godlewski T, Karabenick RL, Stephens JM, Ullrich T, Rice KC, and Woods JH (2003) Pharmacological characterization of the effects of 3,4methylenedioxymethamphetamine ("ecstasy") and its enantiomers on lethality, core temperature, and locomotor activity in singly housed and crowded mice. Psychopharmacology 166:202-211.

Fantegrossi WE, Kiessel CL, De la Garza R 2nd, and Woods JH (2005) Serotonin synthesis inhibition reveals distinct mechanisms of action for MDMA and its enantiomers in the mouse. Psychopharmacology 181:529-536.

Fitzgerald RL, Blanke RV, Glennon RA, Yousif MY, Rosecrans JA, and Poklis A (1989) Determination of 3,4-methylenedioxyamphetamine and 3,4-methylenedioxymethamphetamine enantiomers in whole blood. J Chromatogr 490:5969.

Glennon RA (1989) Stimulus properties of hallucinogenic phenalkylamines and related designer drugs: formulation of structure-activity relationships. NIDA Res Monogr 94:43-67.

Glennon RA, Little PJ, Rosecrans JA, and Yousif M (1987) The effect of MDMA ("Ecstasy") and its optical isomers on schedule-controlled responding in mice. Pharmacol Biochem Behav 26:425-426.

Glennon RA, Titeler M, and Young R (1986) Structure-activity relationships and mechanism of action of hallucinogenic agents based on drug discrimination and radioligand binding studies. Psychopharmacol Bull 22:953-958.

Glennon RA, Young R, Rosecrans JA, and Anderson GM (1982) Discriminative stimulus properties of MDA analogs. Biol Psychiatry 17:807-814.

Goodwin AK and Baker LE (2000) A three-choice discrimination procedure dissociates the discriminative stimulus effects of $d$-amphetamine and ( \pm )-MDMA in rats. Exp Clin Psychopharmacol 8:415-423.

Herin DV, Liu S, Ullrich T, Rice KC, and Cunningham KA (2005) Role of the serotonin 5-HT2A receptor in the hyperlocomotive and hyperthermic effects of (+)-3,4-methylenedioxymethamphetamine. Psychopharmacology 178:505-513.

Institute of Laboratory Animal Resources (1996) Guide for the Care and Use of Laboratory Animals, 7th ed, Institute of Laboratory Animal Resources, Commission on Life Sciences, National Research Council, Washington, DC.

Johanson CE, Kilbey M, Gatchalian K, and Tancer M (2006) Discriminative stimulus effects of 3,4-methylenedioxymethamphetamine (MDMA) in humans trained to discriminate among $d$-amphetamine, meta-chlorophenylpiperazine and placebo. Drug Alcohol Depend 81:27-36.

Kamien JB, Johanson CE, Schuster CR, and Woolverton WL (1986) The effects of $( \pm)$-methylenedioxymethamphetamine and $( \pm)$-methylenedioxyamphetamine in monkeys trained to discriminate (+)-amphetamine from saline. Drug Alcohol Depend 18:139-147.

Koek W, Carter LP, Wu H, Coop A, and France CP (2006) Discriminative stimulus effects of flumazenil: perceptual masking by baclofen, and lack of substitution with gamma-hydroxybutyrate and its precursors 1,4-butanediol and gammabutyrolactone. Behav Pharmacol 17:239-247.

Li JX, McMahon LR, Gerak LR, Becker GL, and France CP (2008) Interactions between delta(9)-tetrahydrocannabinol and mu opioid receptor agonists in rhesus monkeys: discrimination and antinociception. Psychopharmacology 199:199-208. Lim HK, Su Z, and Foltz RL (1993) Stereoselective disposition: enantioselective quantitation of 3,4-(methylenedioxy) methamphetamine and three of its metabolites by gas chromatography/electron capture negative ion chemical ionization mass spectrometry. Biol Mass Spectrom 22:403-411.

Mechan A, Yuan J, Hatzidimitriou G, Irvine RJ, McCann UD, and Ricaurte GA (2006) Pharmacokinetic profile of single and repeated oral doses of MDMA in squirrel monkeys: relationship to lasting effects on brain serotonin neurons. Neuropsychopharmacology 31:339-350.

Mueller M, Peters FT, Maurer HH, McCann UD, and Ricaurte GA (2008) Nonlinear pharmacokinetics of $( \pm) 3,4$-methylenedioxymethamphetamine (MDMA, "Ecstasy") and its major metabolites in squirrel monkeys at plasma concentrations of MDMA that develop after typical psychoactive doses. J Pharmacol Exp Ther 327:38-44.

Nichols DE (1986) Differences between the mechanism of action of MDMA, MBDB, and the classic hallucinogens: identification of a new therapeutic class: entactogens. J Psychoactive Drugs 18:305-313.

Nichols DE and Oberlender R (1990) Structure-activity relationships of MDMA and related compounds: a new class of psychoactive drugs? Ann N Y Acad Sci 600: 613-623; discussion 623-625.

Oberlender R and Nichols DE (1988) Drug discrimination studies with MDMA and amphetamine. Psychopharmacology 95:71-76.

Ortuño J, Pizarro N, Farré M, Mas M, Segura J, Camí J, Brenneisen R, and de la Torre R (1999) Quantification of 3,4-methylenedioxymetamphetamine and its 
metabolites in plasma and urine by gas chromatography with nitrogen-phosphorus detection. J Chromatogr B Biomed Sci Appl 723:221-232.

Partilla JS, Dempsey AG, Nagpal AS, Blough BE, Baumann MH, and Rothman RB (2006) Interaction of amphetamines and related compounds at the vesicular monoamine transporter. J Pharmacol Exp Ther 319:237-246.

Pizarro N, Llebaria A, Cano S, Joglar J, Farré M, Segura J, and de la Torre R (2003) Stereochemical analysis of 3,4-methylenedioxymethamphetamine and its main metabolites by gas chromatography/mass spectrometry. Rapid Commun Mass Spectrom 17:330-336.

Reist M, Carrupt PA, Francotte E, and Testa B (1998) Chiral inversion and hydrolysis of thalidomide: mechanisms and catalysis by bases and serum albumin, and chiral stability of teratogenic metabolites. Chem Res Toxicol 11:1521-1528.

Schechter MD (1987) MDMA as a discriminative stimulus: isomeric comparisons. Pharmacol Biochem Behav 27:41-44.
Wegel RL and Lane CE (1924) The auditory masking of one pure tone by another and its probable relation to the dynamics of the inner ear. Physiol Rev 23:266-285.

Yarosh HL, Katz EB, Coop A, and Fantegrossi WE (2007) MDMA-like behavioral effects of N-substituted piperazines in the mouse. Pharmacol Biochem Behav 88:18-27.

Young R and Glennon RA (2008) MDMA ( $N$-methyl-3,4-methylenedioxyamphetamine) and its stereoisomers: similarities and differences in behavioral effects in an automated activity apparatus in mice. Pharmacol Biochem Behav 88:318-331.

Address correspondence to: Dr. William E. Fantegrossi, University of Arkansas for Medical Sciences, College of Medicine, Department of Pharmacology and Toxicology, 4301 West Markham Street, \#638, Little Rock, AR 72207. E-mail: wefantegrossi@uams.edu 